\title{
The Research on Geographic Cognitive Model and Expressional Model of Cyberspace
}

\author{
Xiao-Hui Chen ${ }^{\text {a, b* }}{ }^{*}$ Feng Li ${ }^{\text {a }}$, Wei Zhang ${ }^{a}$, Bing-Chuan Jiang ${ }^{a}$, and \\ Yu-Ting Liao ${ }^{a}$ \\ a Institute of Zhengzhou Surveying and Mapping, Zhengzhou 450052, China \\ b State Key Laboratory of Geo-information Engineering, Xi-an 710000, China \\ *Corresponding author: Xiao-Hui Chen, cxh_vrlab@163.com
}

\begin{abstract}
The issue is aimed at the demand of cognizant Cyberspace, analyzed the spatial and temporal characteristics of Cyberspace. By means of researching the mapping mechanism between cyberspace and geo-space, constructs the theories and geographical cognitive models of Cyberspace. Based on the theory of cartography, builds the mapping model of cyberspace. Through the visualization algorithm based on geo-space, realizes the visualization of cyberspace and provides the basis of theory and technical support of cognizing and analysis Cyberspace.
\end{abstract}

Keywords: cyberspace, cognitive mode, mapping model

\section{Introduction}

Communication technology and Internet technology has changed human activity patterns. To a certain extent, human interaction has broken through the constraints of time and space. The transmission of information and communication liberate from the constraints of time and Geographic space. Human activities extend from geo-space to the virtual cyberspace. The whole world has been a form of an interdependent giant online world. The fields of politic, economy and military has related to the network which the national infrastructure depends on. Human beings create a new space which is not restricted by region and are parallel to the Geo-space ${ }^{1}$.Many universities in the world have set up the institute of cyberspace. The international cyberspace conference has been a year at once. In 2013, the conference participants reached 10 thousand and the theme is that the open and secure cyberspace realizes global prosperity. The research content is involved in the economic, social security and other issues of cyberspace 。

In conclusion, cyberspace is becoming an important space of human activity whether in people's daily life or on the military and national security. The status of cyberspace is as the Geo-space. Cyberspace is not fixed in the traditional sense of the concept of physical space, and has essentially different from the Geo-space, the object and scope of activities have been beyond the scope of the common cognitive ${ }^{2}$. On the other hand, Cyberspace is closely related, interdependence and common development with the Geo-space, it is more complex and general than the geographical environment. How to cognize and express is a very urgent problem.

\section{Related work}

Geography scholars tried to study the spatial model of cyberspace from the perspective of geography ${ }^{3,4}$. Morley and Robin put forward that the cyberspace provides people with a new type of social space. Cyberspace is a new place for communicating with each other and it is very different from traditional geographic space. 
So far, the model of cyberspace is not perfect. It is short of a suitable and widely accepted model describing the cyberspace. There are three main basic points for depicting the Cyberspace:

\subsection{The view of three-dimensional space}

Benedik $^{5}$ put forward the view of a three-dimensional space which is similar to the geographic space. It considers the cyberspace as a $3 \mathrm{~d}$ coordinate space, each information point has a corresponding $(\mathrm{X}, \mathrm{Y}, \mathrm{Z}$ ) coordinates. Using this model to describe cyberspace, it is limited to information content and easy to make people trek. The view cannot performance the characteristics of the zero time and zero distance in cyberspace.

\subsection{The view of network space}

Luc Girardin ${ }^{6}$ consider the cyberspace as a network space. In the space, computer and information are linked together as a network through a variety of medium. On the concept, node and chain are two basic objects, which set up the model of network topology. It is a kind of widely accepted model which can describe the physical structure of the network diagram and also reflect the relationship between the information. But this model has a problem is that it cannot comprehensively express the various phenomena of the cyberspace by a limited amount of information.

\subsection{The view of information space}

Staple think from the viewpoint of information space which can solve the long-standing problem how to draw the multidimensional objects to the $2 \mathrm{~d}$ drawings. This view ignored the problem existing in the integrity of the relationships in cyberspace. It was only focus on the model of the local information, but missing the information infrastructure which is the important constitutes of cyberspace.

\section{Based on geographic information building the cognitive model of Cyberspace}

This article uses the Staple's point of view, from the viewpoint of information space, considers the factor of geographical information building cognitive model of cyberspace based on the geographic information. It adopts a triple formalization to describe the space model $G$ of cyberspace, $G$ is a formal triple:

$$
\begin{gathered}
\mathrm{G}=(\mathrm{V}, \mathrm{E}, \mathrm{T}) \\
\mathrm{V}=\{\mathrm{v} \mid \mathrm{v} \in \text { node }\} \\
\mathrm{E}=\left\{\left(\mathrm{v}_{0}, \mathrm{v}_{1}\right) \mid \mathrm{v}_{0} \in \mathrm{V} \wedge \mathrm{v}_{1} \in \mathrm{V}\right\} \\
\mathrm{T}=\{(\mathrm{x}, \mathrm{y}) \mid(\mathrm{x}, \mathrm{y}) \in \text { Geographical spatial coordinates }\},
\end{gathered}
$$

\subsection{The model of node}

Node mainly refers to the abstract as a node in network model, which forms the core of the whole network model. The node-class is used to describe the various nodes of cyber space, such as the location of the nodes, the IP information, type, etc. The information visualization system reads the IP of node, draws the network topology and the status while finding and connecting the nodes.

\subsection{The model of link}


In the network model, it composed of the correlation between the nodes by wired and wireless communication links, but the state of the node would impact on the state of the link directly or indirectly. Link is used to describe the link information in the network connection, such as link connection node, link of visual way, type, etc. Information visualization is the main task of the network link and correct flow in all kinds of information on the link.

\section{Based on geographic information building the cognitive model of Cyberspace}

In modern cartography, map is defined as: according to certain mathematical laws, using the drawing language, through the cartographic generalization, on a certain carrier, express all kinds of things on the earth (or other object) spatial distribution, contact time and pattern of the development and changes in the state. In summary, the three elements of a map are "mathematical laws, map language, cartographic generalization".

\subsection{The mathematical basis of Cyberspace}

It is according to the related concepts of graph theory, through the research of network model, constructing cyberspace metric system. Generally, the network is described as structure model consists of node set V and edge set E. Usually, we express the node by a circular, square or rectangle, the edge by straight line or curve. It can use the graph $G=(V, E)$ expressing the visualization of Network, in which $\mathrm{V}$ is a non-empty set, $\mathrm{E}$ is $\mathrm{V}$ elements in disorder or the set of ordered pair, and use the $|\mathrm{V}|$ and $\mid \mathrm{E}$ |expressing the number of nodes and edges.

The information in the graph $G$ can use the Matrix to express: Adjacent Matrix. Adjacent matrix of the graph G: $S=S(G)$ is the $n * n$ matrix. Variable is on behalf of adjacency relations between the two nodes:

$$
\mathrm{S}_{i j}=\left\{\begin{array}{cc}
1 & \text { If } \mathrm{V}_{i} \text { and } \mathrm{V}_{j} \text { is adjacent } \\
0 & \text { If } \mathrm{V}_{i} \text { and } \mathrm{V}_{j} \text { is not adjacent }
\end{array}\right\}
$$

The oblique diagonal variable is not defined, because it is not allowed any loop in the graph G.

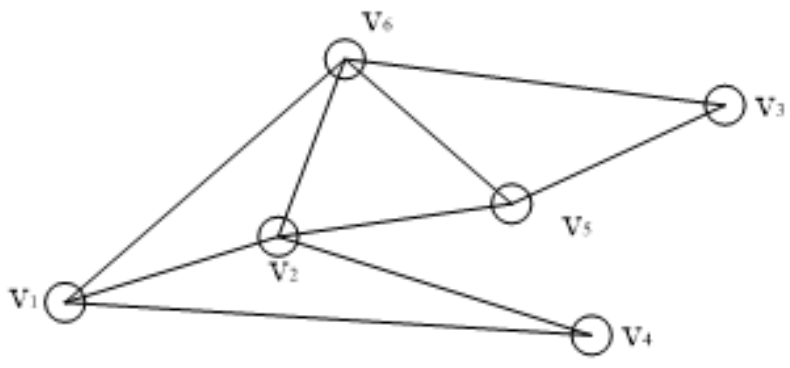

$$
S=\left(\begin{array}{cccccc}
- & 1 & 0 & 1 & 0 & 1 \\
1 & - & 0 & 1 & 1 & 1 \\
0 & 0 & - & 0 & 1 & 1 \\
1 & 1 & 0 & - & 0 & 0 \\
1 & 1 & 1 & 0 & - & 1 \\
1 & 1 & 1 & 0 & 1 & -
\end{array}\right)
$$

Fig.1-Graph G and its Adjacent Matrix

The following parameters are used to extract the features and contents of graph $\mathrm{G}$, and related parameters in the network measurement model:

(1) Distance measure

In real geographical space, the distance of two entities is the Euclidean distance. Euclidean distance is the distance of the best-known measure. In n-dimensional Euclidean space, each point is a n-d real vector. The traditional distance measure is defined as follows:

$$
\mathrm{d}\left(\left[x_{1}, x_{2}, \cdots, x_{n}\right],\left[y_{1}, y_{2}, \cdots, y_{n}\right]\right)=\sqrt{\sum_{i=1}^{n}\left(x_{i}-y_{i}\right)^{2}}
$$


Cyberspace is a space in which it uses the network system and related physical infrastructure, electrical and electromagnetic spectrum to storage, modify, and exchange data. On the one hand, the cyberspace is a real physical space. On the other hand, the cyberspace is a virtual information space, in this case, the distance between the objects using the network distance than the Euclidean distance is more conform to the requirements. The distance of node $\mathrm{i}$ and node $\mathrm{j}$ is defined as the shortest path connecting the two nodes on the number of edges. The maximum distance between any two nodes in a network is referred to as the diameter of the network, named D:

$$
\mathrm{D}=\max _{i, j} \mathrm{~d}_{i j}
$$

The definition of the average path length is the average of the distance between any two nodes:

$$
\mathrm{L}=\frac{1}{\frac{1}{2} N(N+1)} \sum d_{i j}
$$

(The $\mathrm{N}$ is the number of the network nodes)

In conclusion, cyberspace is not a substantive space. So, it is difficult to measure by any substantive measured indicators and the continuity of time and space. The traditional Euclidean distance measure is not applicable. Plewe (1995) defined the other three distances in addition to the physical distance: the first is an effective distance. The second is psychological distance. The third is the overall distance. Cyberspace distance belongs to the first one: the effective distance. Therefore, this paper uses the complex network model of distance measurement as the distance of the cyberspace, it is more according with the actual demand of cyberspace.

(2) Spatial relation measure

Cyberspace has broken the limitation of distance which can't describe the strength of the correlation degree between cyberspace entities. It needs to find a new measurement scale to define the correlation between things. Nodes and edges are the most basic elements of a constitution in cyberspace where the transmission of information is a connectivity. And, the relationship between the node and edge is the only topological relationship. The more number of edges means the more connections between nodes and the stronger of the correlation. Therefore, from the perspective of probability and statistics, we can use the distribution to determine the spatial relationship of cyberspace.

(3) Spatial distribution measure

In Cyberspace, it uses relations as a representative of the new characteristics instead of traditional spatial relationship expression which is the distance and direction. Aggregation coefficient is specially used to measure the parameters of the network node clustering. The network clustering coefficient is all nodes cluster coefficient of average. Coefficient can use small world model gathered the closer:

Using $\operatorname{deg}(\mathrm{v})$ represents the node degree, $|\mathrm{E}(\mathrm{G}(\mathrm{v}))|$ represents the number of connections between all adjacent nodes, then the clustering parameter is as follows:

$$
\mathrm{C}(\mathrm{v})=\frac{2|\mathrm{E}(\mathrm{G}(\mathrm{v}))|}{\operatorname{deg}(\mathrm{v}) \times(\operatorname{deg}(\mathrm{v})-1)}
$$

In nearly a decade, people researched by a large number of empirical studies and statistical analysis, confirmed that the network which is the constitution of cyberspace generally has 
high aggregation, the shorter average distance, the geometric characteristics of power law and exponential distribution.

Above all, to measure the statistical parameters including: the total number of nodes, the total number of edges, average degree, average path length, clustering coefficient; among them, the degree, path length and distance coefficient are the most important elements.

\subsection{The map language of Cyberspace}

The elements of Cyber-map can be described in a certain form of informational infrastructure and physical parts such as electronic equipment. It also can be described as the form of abstract information, data and network information such as attribute part. It needs to represent the qualitative features and quantitative characteristics, so the cyber language symbols are different from the traditional map. It uses a large number of abstract geometry symbols and the most commonly used symbols are the point symbol and the line symbols, individual map expressing the network clustering information will use planar symbols.

(1) Point symbol

The point symbol of Cyber-map is mainly used to represent the network nodes which can be a physical infrastructure, also can be a certain information point. For example, it would be the topology of network physical structure of the equipment, or different web pages in the web site, or a hypertext document, etc. The shape and colour is the most important visual elements of qualitative representation and classification variables, while size can represent quantitative characteristics in cyber-map.

(2) Line symbol

The line symbol of Cyber-map mainly is mainly used to express the links between nodes. Namely, whether there is a relationship between nodes. When show the cyberspace physical structure, it uses the different thickness to represent the bandwidth, the colour different to represent the flow, the different shapes to represent the connection way. For the description part, line symbol just means a qualitative relationship.

(3) Planar symbol

The planar symbol of Cyber-map is mainly used to express one or several common attribute information in the network, which is used to distinguish between different kinds of information. The colour and design are used to show the elements of qualitative and classification.

\subsection{Multi-scale expression of cyberspace}

Cyberspace is a combination of physical domain and virtual information domain. It is also a combination of concrete and the abstract. From the virtual information domain, it can construct a virtual world which is similar to the geographic world completely through different forms of digital interaction and communication. It also can create thousands of rapidly expanding or shrinking fast great space and individual character different space. That makes the multi-scale expression of cyberspace is extremely difficultly. It is a problem which is very hard to solve in cyber-map expression. In many instances, most cyber-maps are the structure of network topology, where the multi-scale expression of cyber-map mainly involves comprehensive of the node and the link. This paper will apply the group structure detection algorithm to multi-scale expression, using the structure detection means to realize the multi-scale expression of cyberspace.

(1) The generalization of node 
The generalization of node mainly involves of the node selection, summary and displacement three basic forms. Node as one of the most important elements in cyber-map can represent both the physical part of the physical infrastructure in cyberspace (such as servers, routers, etc.), and the part of the information (such as website, text, etc.). At the same time, it can express a single node, but also a collection of multiple nodes. On account of the node important degree, the restrictions of the display area and the difference of map presentation content, it is necessary to select a certain node. And some of the nodes have the same properties, sometimes may occur stacking, etc. At this moment, it needs to summarize and displace the nodes. The process of operation and displacement nodes is based on the multiscale expression of related principles in the traditional cartography.

(2) The generalization of link

The generalization of link mainly involves the link selection, reduction, summary and displacement four basic forms. Selection is based on the node of a link, in the selection of nodes at the same time for the selection of link. And the links between cross each other often occur, and some are very close and have the same properties, this is where the reduction and summary are carried out on the link. Meanwhile, for making the map look more clearly, it also needs to be displacement, etc.

As shown, in figure 2 is nodes topology in the cyberspace. Using the detection algorithm with group structure, it found three group structures, as shown in figure 2. According to the testing results of the group structure, it integrated network nodes and links, as shown in figure 2.
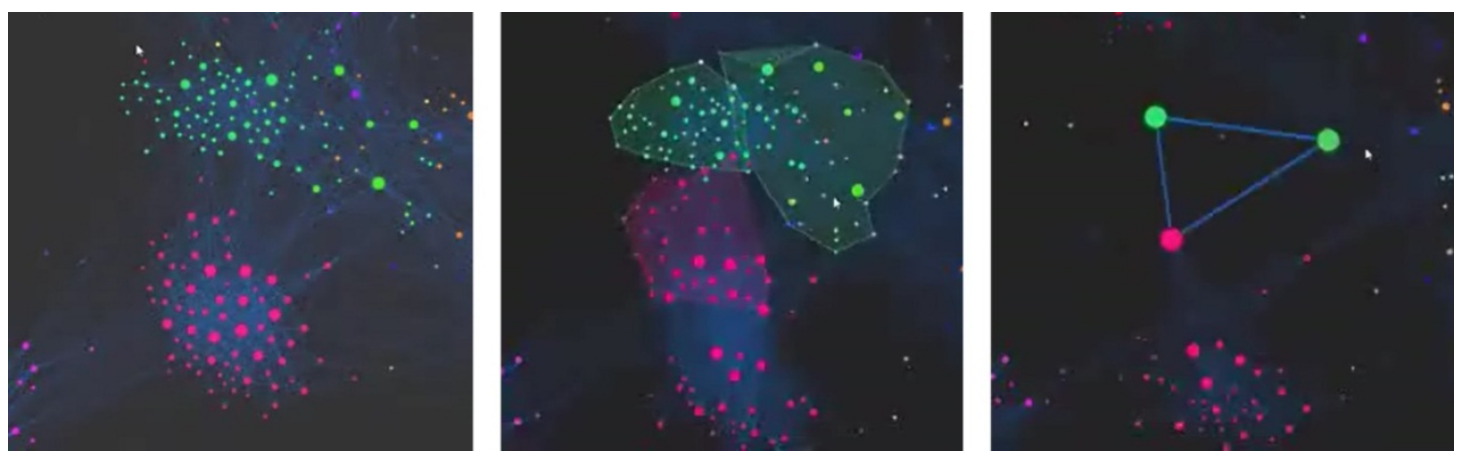

Fig.2-The multi-scale expression of cyberspace

\section{Conclusion}

The current research on cyberspace causes much attention around the world, and cyberspace for geography, is a relatively new research field. Many international and domestic discussion for this often covers only the one aspect on the one hand, it has been in the exploratory stage. From cognitive cyberspace geography, this paper studies the intersection of information science, complex network science, geography. For cognizing cyberspace as the starting point, this paper builds the geography model of cyberspace and the cartographic model of cybermap, realizes the visualization of cyberspace.

\section{Acknowledgements}

This thesis is supported by State Key Laboratory of Geo-information Engineering, NO SKLGIE2014-Z-4-1 and NO SKLGIE2015-Z-4-1 and the National Natural Science Foundation of China (41301428) 


\section{References / Literatura}

1. Z. Zhan, The research on construction theories of Cyber-map [D]. Information University Master's thesis, 2012

2. $\quad Y$. Chen, $J$ Jin., Innovation of the methods of geographical researches and its future [J].Arid Land Geography, 2003, 26 (2): 97-102.

3. AL Barabási, R Albert, H Jeong, Scale-free characteristics of random networks: the topology of the world-wide web [J]. Physica A: Statistical Mechanics and its Applications, 2000, 281 (1): 69-77

4. URL: http://www.cybergeography.org.

5. M.Benedik, Cyberspace: First Steps[J]. The MIT Press, Cambring, Massachusettes, London. 1992

6. Girardin. Mapping virtual locations to avoid the lost-in-cyberspace syndrome[Z].1995 